(median: 2304, IQR: 1519-2723. $\mathrm{p}=0.0238, \mathrm{p}=0.038$ respectively) (figure 1).

Conclusion Eosinophils in COPD show enhanced migration towards IL-5 and eotaxin. Whether this is a mechanism underlying T2 high COPD requires further investigation.

\section{P55 EXPLORING RHINOVIRUS-INDUCED ER STRESS IN BRONCHIAL AIRWAY EPITHELIAL CELLS}

KL Bradley, CA Stokes, LC Parker, AM Condliffe. The University of Sheffield, Sheffield, UK

10.1136/thoraxjnl-2017-210983.197

Rational and Hypothesis Human Rhinovirus (HRV) infections are major contributors to the increased morbidity burden associated with asthma and COPD acute exacerbations. There are currently no effective treatments or vaccines targeting exacerbations, therefore understanding the host-virus interactions that drive cellular damage will help identify potential therapeutic targets. Viral infections alter the airway environment through increased production of inflammatory mediators, defensive factors and viral proteins. This Results in the upregulation of cellular processes such as the unfolded protein response (UPR), an ER (endoplasmic reticulum) stress pathway that acts to alleviate ER stress caused by increased demands on protein synthesis. In the event that UPR fails to restore cellular homeostasis, pro-apoptotic pathways are activated. Many viruses induce ER stress and have evolved mechanisms to modify UPR to promote their own replication. Interestingly, the mechanisms and consequences of HRV-induced ER stress in bronchial epithelial cells have yet to be explored. We therefore hypothesised that HRV infection induces and manipulates ER stress processes within bronchial epithelial cells.

Objectives To explore the mechanisms and consequences of HRV-induced ER stress within bronchial epithelial cells.

Methods The immortalised bronchial epithelial cell line, BEAS2B was infected with HRV for 1 hour at MOI 1.5. Induction and subcellular localisation of ER stress markers (GRP78 and ATF4) were measured at various time points by western blotting and confocal microscopy. Tunicamycin (a known ER stress inducer) and filtered HRV were included as positive and negative controls respectively.

Findings Virally infected BEAS-2B cells induced ER stress as evidenced by the significant induction of the UPR chaperone protein, GRP78 at 24 hour. ATF4, a transcriptional activator of UPR target genes, redistributed from a cytoplasmic location to perinuclear regions, as assessed by immunofluorescence and confocal microscopy. Translocation was seen from as early as 1 hour following treatment with Tunicamycin, but this response was relatively delayed in HRV-infected BEAS-2B cells, with ATF4 redistributing to perinuclear regions from 8 hour post infection.

Conclusion Our data demonstrate for the first time HRVinduced ER stress within bronchial epithelial cells, and suggest that HRV may manipulate ER stress pathways to facilitate its own replication.

\section{P56 HUMAN RHINOVIRUS IMPAIRS PHAGOCYTOSIS OF HAEMOPHILUS INFLUENZAE IN ALVEOLAR MACROPHAGES IN CHRONIC OBSTRUCTIVE PULMONARY DISEASE}

UJ Finney, K Belchamber, S Kemp, G Donaldson, P Mallia, SL Johnston, JA Wedzicha. Imperial College, London, UK

10.1136/thoraxjnl-2017-210983.198

Introduction COPD exacerbations are the main cause of hospital admission and death in COPD. Respiratory viruses are identified in over half COPD exacerbations with human rhinovirus (HRV) being the most commonly detected. Secondary bacterial infection is associated with prolonged exacerbations, higher rates of hospital admission and increased symptom severity. Our group have previously shown that secondary bacterial infection in HRV induced COPD exacerbations is driven by an outgrowth of Haemophilus influenzae.

Hypothesis We hypothesised that HRV may impair phagocytosis of bacteria by alveolar macrophages which may lead to secondary bacterial outgrowth in COPD exacerbations.

Methods Bronchoscopy was performed on participants of the London COPD cohort and healthy controls. Alveolar macrophages were obtained by bronchoalveolar lavage. Alveolar macrophages were incubated with $\mathrm{HRV}$ at a multiplicity of infection (MOI) of 5 for 24 hours or media control. Phagocytic capacity was assessed by incubating with fluorescently labelled heat killed Haemophilus influenzae or Streptococcus pneumoniae for 4 hours. Uptake was measured in Relative Fluorescent Units (RFU) using a fluorimeter.

Results Alveolar macrophages were obtained from 14 COPD patients and 9 healthy controls. HRV significantly impaired phagocytosis of $H$. influenzae by alveolar macrophages in patients with COPD (HRV median 0.97 (0.50-2.17 interquartile range) RFU $\times 10^{3}$ vs media control median $1.38(0.70$ 2.50 interquartile range) RFU $\times 10^{3} \mathrm{p}<0.05$ ) but did not impair phagocytosis of $S$. pneumoniae. HRV did not impair phagocytosis in alveolar macrophages from healthy controls. Baseline phagocytic capacity of $\mathrm{H}$. influenzae was impaired in COPD patients compared to healthy controls (COPD 1.59+/1.31 RFU $\times 10^{3}$ vs healthy control $3.81+/-1.82$ RFU $\left.\times 10^{3}\right)$. Phagocytosis of $\mathrm{H}$. influenzae correlated with worsening FEV1 percent predicted in COPD $\left(\mathrm{R}^{2}=0.452 \mathrm{p}<0.05\right)$.

Conclusions The presence of HRV impaired phagocytosis of $H$. influenzae in alveolar macrophages from patients with COPD but not healthy controls. This may contribute to secondary bacterial infection in COPD exacerbations.

\section{P57 SOLUBLE RECEPTOR FOR ADVANCED GLYCATION END- PRODUCTS (SRAGE) IN PATIENTS WITH COPD: THE ERICA STUDY}

R Siraj, TM McKeever, L Buss, D Mohan, K Maki-Petaja, J Forman, CM McEniery, J Cheryian, N Gale, JR Cockcroft, PM Calverley, W MacNee, B Miller, R Tal-Singer, M Polkey, IB Wilkinson, CE Bolton. The University of Nottingham, Nottingham, UK

10.1136/thoraxjnl-2017-210983.199 